\title{
Ein Mixed-Methods-Design zur Erforschung "sozialer« Armut
}

Die in dieser Arbeit verwendeten Daten stammen aus dem Forschungsprojekt „Aspekte der Armut in Mecklenburg-Vorpommern“, das im Auftrag der Arbeiterwohlfahrt, Landesverband Mecklenburg-Vorpommern, von Forscher*innen der Universität Greifswald, der Hochschule Neubrandenburg, der Universität Rostock und des Thünen-Instituts für Regionalentwicklung e. V. durchgeführt wurde (AWO Landesverband Mecklenburg-Vorpommern e. V., 2015). Ziel der Studie war es, ein Beispiel dafür zu geben, wie eine Armuts- und Sozialberichterstattung für das Land Mecklenburg-Vorpommern aussehen könnte. Neben der Bündelung und Systematisierung sozialstatistischer (Hochschule Neubrandenburg) und demographischer Daten (Universität Greifswald) war es Aufgabe der Forscher*innen, Lebenslagen von Menschen in relativer Einkommensarmut (Einkommen unter $60 \%$ des Landesmedians) zu rekonstruieren (Universität Rostock, Thünen-Institut für Regionalentwicklung e. V.). Dazu wurden unter Mitarbeit des Autors dieser Arbeit 49 problemzentrierte Interviews (Witzel \& Reiter, 2012) mit von Armut betroffenen Menschen aus städtischen und ländlichen Räumen Mecklenburg-Vorpommerns geführt und ausgewertet (Klärner et al., 2015) ${ }^{1}$. Das Sample wurde nach Abschluss der Studie nochmals erweitert durch die Befragung einer Kontrastgruppe von acht Studierenden in relativer Einkommensarmut. Insgesamt setzt sich der Datensatz also aus 57 Befragten zusammen. In Ergänzung zum qualitativen Interview wurden die egozentrierten Beziehungsnetzwerke der Befragten mithilfe eines standardisierten Fragebogens unter Anwendung der Software VennMaker (Gamper et al., 2012) erhoben.

\footnotetext{
${ }^{1}$ In der Studie selbst wurden 33 Einzelinterviews in der Stadt geführt und 16 Einzelinterviews im ländlichen Raum. Zwei weitere Fallbetrachtungen aus der Studie werden hier nicht analysiert, da die Datengrundlage in diesen Fällen zu sehr von den übrigen Interviews abweicht (1 Gruppeninterview sowie eine teilnehmende Beobachtung).
} 
Durch die Integration von standardisierten und nicht-standardisierten Verfahren im Rahmen der Datenerhebung und -analyse handelt es sich um ein Mixed-Methods-Design. Mixed Methods Designs unterscheiden sich nach der Art und Weise der Integration der verschiedenen Paradigmen, der Phase, in der die Zusammenführung der qualitativen und quantitativen Daten erfolgt, und der Gewichtung zwischen den Anteilen der beiden Datenarten (Creswell 2010, Hollstein 2014, S. 12) ${ }^{2}$. In diesem Fall handelt es sich um ein weitgehend paralleles Design mit zwei getrennt voneinander erhobenen und analysierten Datensätzen, die erst am Ende der Analyse aufeinander bezogen werden.

Zunächst werden in diesem Kapitel die Befragungsinstrumente und die Vorgehensweise bei der Datenerhebung vorgestellt, um darauf aufbauend die Schritte der Auswertung der so gewonnenen Daten zu beschreiben. Zum Abschluss des Kapitels erfolgt eine Reflexion zum Verhältnis von standardisiert erhobenen Netzwerkdaten $\mathrm{zu}$ qualitativem Interviewmaterial und $\mathrm{zu}$ den Möglichkeiten und Grenzen der Integration dieser beiden Ansätze.

\subsection{Datenerhebung}

Im Folgenden werden die Besonderheiten der Untersuchungsregion (Abschnitt 4.1.1), die Zusammensetzung des Samples (4.1.2) und die verwendeten Erhebungsinstrumente erläutert. Dazu zählen der Leitfaden zum problemzentrierten Interview (4.1.3), die quantitative Netzwerkerheung (4.1.4) und der soziodemographische Datenbogen (4.1.5).

\subsubsection{Untersuchungsregion und Zielgruppe}

Mecklenburg-Vorpommern gilt als besonders strukturschwaches, weitgehend ländliches Bundesland mit einer sehr geringen Bevölkerungsdichte (Steinführer et al., 2016). Das Land war spätestens seit dem Beitritt der DDR zur BRD von selektiver Abwanderung geprägt, die zu einem bis heute anhaltenden Männerüberschuss in den mittleren Altersstufen führte (Statistisches Amt

\footnotetext{
${ }^{2}$ Sequentielle Designs führen die verschiedenen Ansätze sukzessive aufeinander aufbauend ein, während bei parallelen Designs beide Datenarten gemeinsam erhoben und in der Regel zunächst einmal getrennt voneinander analysiert werden (Hollstein 2014, S. 14 f.). Schließlich gibt es noch Fully Integrated Designs, bei denen eine wechselseitige Bezugnahme beider Daten- und Analysearten während des gesamten Forschungsprozesses vorgenommen wird (ebd. 15 f.).
} 
Mecklenburg-Vorpommern, 2019). Bereits seit Mitte der 1980er Jahre kam es zu Bevölkerungsrückgängen, die sich bis in die erste Hälfte der 2010er Jahre weiter fortsetzten. So sank die Bevölkerungszahl von 1,959 Millionen im Jahr 1985 auf einen Tiefstwert von 1,596 Millionen im Jahr 2013 (ebd. S. 27). Der seit $2013 \mathrm{zu}$ beobachtende positive Wanderungssaldo liegt seitdem häufig über dem hohen Sterbefallüberschuss (2018: 8916 Personen, ebd., S. 24), sodass die Bevölkerungsgröße seitdem wieder leicht zugenommen hat (2018: 1,609 Mio., ebd.).

Diese demographischen Prozesse führten über die Jahrzehnte zu einer deutlichen Veränderung der Altersstruktur: Der Anteil der Bevölkerung unter 18 Jahren sank seit 1990 von 25,4 \% auf 15,2 \% während sich der Anteil der über 65-jährigen von 10,9 \% auf 24,7 \% erhöhte (ebd. S. 28, eigene Berechnung). Die derzeitige Altersstruktur wird aufgrund der hohen Zahl zu erwartender Renteneintritte in den nächsten Jahren zu einer weiteren Abnahme der Anzahl erwerbsfähiger Personen führen. In der 5. Bevölkerungsprognose des Landes Mecklenburg-Vorpommern wird vorhergesagt, dass der Anteil der 18- bis 65jährigen bis 2040 um weitere 18,4 \% sinken könnte, während der Anteil der über 65-jährigen um 28,2 \% steigen könnte (Mecklenburg-Vorpommern, 2019a, S. 13). In Bezug auf die Chancen zur Überwindung relativer Einkommensarmut durch Erwerbsarbeit kann das durchaus positive Auswirkungen auf die Betroffenen haben (sinkende Erwerbslosigkeit). Zunehmen wird dagegen die Altersarmut, da in den nächsten Jahren die Renteneintritte jener Kohorten bevorstehen, die seit 1990 stark von Erwerbslosigkeit und prekärer Beschäftigung betroffen waren. Ihre Rentenanwartschaften werden daher niedriger ausfallen als die der vorangegangenen Kohorten.

Innerhalb des Bundeslandes Mecklenburg-Vorpommern gibt es jedoch eine erhebliche Heterogenität, die sich ebenfalls bereits an den demographischen Faktoren ablesen lässt. So geht die regionalisierte Bevölkerungsprognose des Landes davon aus, dass die Städte Rostock und Schwerin bis 2040 um 7,8 \% bzw. um 3,2 \% wachsen werden, während die ländlichen Großkreise moderate bis drastische Schrumpfungen von 0,5\% im Landkreis Rostock bis zu $13 \%$ im Landkreis Mecklenburgische Seenplatte zu erwarten hätten (MecklenburgVorpommern, 2019b, S. 4). In den vergangenen Jahren wurden als Reaktion auf die abnehmende Bevölkerungsdichte Kreisgebietsreformen durchgeführt, die zwar dünn besiedelte, dafür aber flächenmäßig sehr große Landkreise hervorbrachten. Deren Bewohner*innen müssen teilweise sehr weite Strecken auf sich nehmen, um die zentralisierten Daseinsvorsorgeeinrichtungen zu erreichen. Es liegt auf der Hand, dass sich die Situation in diesen sehr ländlichen Regionen stark von jener in den größeren Städten des Landes unterscheidet. 
Die regionale Armutsgefährdungsquote (also der Anteil der Haushalte, deren Äquivalenzeinkommen unterhalb von $60 \%$ des mittleren Einkommens lag) betrug zum Befragungszeitpunkt im Jahr 2015 in den Raumordnungsregionen Mecklenburg-Vorpommerns zwischen 12,9 \% (jeweils Mecklenburgische Seenplatte und Westmecklenburg) und 14,3 \% (Mittleres Mecklenburg/Rostock) und lag damit unter dem bundesweiten Wert von 15,7 \% (amtlichesozialberichterstattung.de ${ }^{3}$ ). Gemessen am Bundesmedian lag die Armutsgefährdungsquote auf Landesebene jedoch bei $21,7 \%$ und damit deutlich über dem bundesweiten Durchschnitt (ebd.). Interessanterweise lag die Einkommensreichtumsquote 2015 (Anteil der Einkommen ab einer Höhe von mehr als $200 \%$ des mittleren Einkommens) sowohl gemessen am Bundesmedian (2,9 \%) als auch am Landesmedian (5,6 \%) deutlich unter dem bundesweiten Wert von $8,2 \%$. Wir haben es in Mecklenburg-Vorpommern also mit einer geringen Ungleichheit auf niedrigem Niveau zu tun: Die Einkommen sind insgesamt niedrig und liegen dicht beieinander.

Während die regionalen Armutsquoten also relativ gering sind, liegt die SGBII-Quote im Moment noch über dem bundesweiten Durchschnitt (2018: 10,8\% in M-V / 8,6 \% im Bund), auch wenn sie sich diesem Niveau - dem Trend der neuen Bundesländer folgend - stetig angleicht (2006: 20,4 \%, 2015: 13,3\%, 2018: $10,8 \%$, ebd.).

Mecklenburg-Vorpommern ist daher eine besonders interessante Region, wenn es darum geht, Transformationsprozesse nach 1990 in ländlichen und städtischen Räumen zu untersuchen. Hier lassen sich Räume vergleichend analysieren, die sich hinsichtlich ihrer ökonomischen, demographischen und siedlungsstrukturellen Gegebenheiten stark voneinander unterscheiden, obwohl sie derselben föderalen Ordnung unterliegen. Die Auswahl dieser transformativen Untersuchungsregion führt dazu, dass makrostrukturelle Zäsuren und Entwicklungen nie ausgeklammert werden können, sondern bei der Analyse des Bewältigungshandelns der Befragten immer mit einbezogen werden müssen. Dazu zählt neben Abwanderung, Schrumpfung und Alterung der Bevölkerung ${ }^{4}$ insbesondere der Umbau der Landwirtschaft vom Agrargenossenschaftlichen LPG-Wesen hin zu maximal automatisierten industriellen Großbetrieben, eine drastische Reduktion des Industriesektors und der Ausbau des Tourismus (Maschke und Köncke, 2020). Damit hat die Region in den letzten 30 Jahren im Schnelldurchlauf Prozesse

\footnotetext{
${ }^{3}$ Alle Werte in diesem Abschnitt wurden abgerufen am: 10.01.2020.

${ }^{4}$ Diese sehr auf die makrostrukturellen Defizite gerichtete Sichtweise kann und sollte in der Forschung nicht unhinterfragt bleiben. Eine Möglichkeit der Differenzierung bietet die Rekonstruktion der Motive von Personen, die M-V dennoch nicht verlassen haben (siehe: Rühmling und Schiemann, 2019; Rühmling 2020).
} 
durchlaufen, die anderen Regionen erst bevorstehen bzw. die man dort mit Blick auf die Erfahrungen aus Nordostdeutschland vielleicht anders gestalten kann.

Um einen möglichst großen Kontrast zu erhalten, wurden die Interviews in Rostock (2018: 1152 Einwohner je $\mathrm{km}^{2}$ bei 208886 Einwohner*innen, Statistisches Amt Mecklenburg-Vorpommern, 2019, S. 26) sowie im Landkreis Mecklenburgische Seenplatte (2018: 47 Einwohner je $\mathrm{km}^{2}$ bei 259130 Einwohner*innen, ebd.) erhoben.

\subsubsection{Rekrutierung und Sample}

Auf Basis von Literaturrecherchen wurden Zielgruppen ausgewählt, die als besonders häufig von relativer Einkommensarmut betroffen gelten: Alleinerziehende, Langzeiterwerbslose, ältere Menschen, Menschen mit Behinderungen oder psychischen Erkrankungen sowie Asylbewerber*innen, Flüchtlinge und andere Personen mit Migrationshintergrund (Klärner et al., 2015, S. 29). Die meisten Befragten wurden in Institutionen rekrutiert, in denen den Forscher*innen die Möglichkeit gewährt wurde, ihr Anliegen vorzutragen. Dazu gehörten Maßnahmen der Jobcenter, Stadtteil- und Begegnungszentren, Migrationsberatungsstellen und Gemeinschaftsunterkünfte für Asylbewerber*innen, Sozialkaufhäuser und Tafeln. Von entscheidender Bedeutung für eine erfolgreiche Rekrutierung zeigte sich dabei die Unterstützung durch die Mitarbeiter*innen der Institutionen. Sie stellten das für eine erfolgreiche Rekrutierung nötige, Vertrauen zwischen Forscher*innen und Interviewpartner*innen her.

Da sich diese Strategie im ländlichen Raum als weniger erfolgreich herausstellte (weniger Einrichtungen und weniger potentielle Interviewpartner*innen, die sich dort aufhalten), wurde hier zusätzlich auch über Annoncen in Anzeigenblättern und auf e-bay-Kleinanzeigen rekrutiert. Gegen eine Aufwandsentschädigung von $20 €$ konnte auf diese Weise ein großer Teil der Interviewpartner*innen in dieser Teilgruppe gewonnen werden.

Die Verteilung der Interviewpartner*innen nach ihren soziodemographischen Eigenschaften ist in Tabelle 4.1 dargestellt. Insgesamt wurden 41 Interviews in der Stadt geführt und 16 Interviews im ländlichen Raum. In beiden Teilgruppen wurden sowohl ältere als auch jüngere Männer und Frauen mit unterschiedlichen Bildungsabschlüssen befragt. Knapp die Hälfte der Befragten aus der Stadt sind erwerbslos oder nehmen an einer Maßnahme des Jobcenters teil (zusammengefasst in der Kategorie ,erwerbslos“), zehn weitere befinden sich in Studium (8) oder Ausbildung (2), neun sind alters- bzw. erwerbsunfähigkeitsberentet und zwei 
der Befragten gehen einer Erwerbstätigkeit nach. Diese Streuung des Erwerbsstatus ließ sich bei der Rekrutierung im ländlichen Raum leider nicht realisieren. Hier ist die überwiegende Mehrheit erwerbslos. Die 6 Befragten ohne deutsche Staatsangehörigkeit lebten zum Zeitpunkt des Interviews ebenfalls alle in der Stadt.

Tabelle 4.1 Soziodemographische Eigenschaften der Interviewpartner*innen

\begin{tabular}{|c|c|c|c|c|}
\hline & & Stadt & Ländlicher Raum & Gesam \\
\hline \multirow[t]{5}{*}{ Alter } & bis 29 & 13 & 4 & 17 \\
\hline & $30-39$ & 11 & 2 & 13 \\
\hline & $40-49$ & 5 & 0 & 5 \\
\hline & $50-59$ & 9 & 7 & 16 \\
\hline & $60+$ & 3 & 3 & 6 \\
\hline \multirow[t]{2}{*}{ Geschlecht } & männlich & 17 & 9 & 26 \\
\hline & weiblich & 24 & 7 & 31 \\
\hline \multirow[t]{3}{*}{ Schulabschluss } & niedrig & 15 & 7 & 22 \\
\hline & mittel & 14 & 6 & 20 \\
\hline & hoch & 12 & 3 & 15 \\
\hline \multirow[t]{6}{*}{ Erwerbsstatus } & erwerbslos & 20 & 14 & 34 \\
\hline & in Ausbildung & 2 & 0 & 2 \\
\hline & EU-Rente & 3 & 0 & 3 \\
\hline & Altersrente & 6 & 1 & 7 \\
\hline & Studium & 8 & 0 & 8 \\
\hline & erwerbstätig & 2 & 1 & 3 \\
\hline \multirow[t]{2}{*}{ Staatsangehörigkeit } & deutsch & 35 & 16 & 51 \\
\hline & nicht-deutsch & 6 & 0 & 6 \\
\hline Gesamt & & 41 & 16 & 57 \\
\hline
\end{tabular}

Wie in Tabelle $4.2 \mathrm{zu}$ sehen ist, konnten Personen aus jeder der oben genannten Zielgruppen rekrutiert werden. In einigen Fällen lassen sich dieselben Personen mehreren Zielgruppen zuordnen (z. B. langzeiterwerbslose Alleinerziehende) weshalb es zu Mehrfachnennungen in der Übersicht kommt.

Durch die breite Streuung der soziodemographischen Eigenschaften und Kontextbedingungen der Befragten bietet das Sample eine geeignete Grundlage zur komparativen Analyse (Glaser \& Strauss, 2010, 53 ff.; Strauss \& Corbin, 1996, 


\section{Tabelle 4.2}

Interviewpartner*innen nach Zielgruppen

\begin{tabular}{l|l}
\hline Zielgruppe & $\begin{array}{l}\text { Anzahl } \\
\text { (Mehrfachnennungen } \\
\text { möglich) }\end{array}$ \\
\hline Alleinerziehende & 7 \\
\hline Langzeiterwerbslose & 34 \\
\hline über 60-jährige & 6 \\
\hline $\begin{array}{l}\text { Menschen mit } \\
\text { Behinderungen }\end{array}$ & 5 \\
\hline Migrant*innen & 6 \\
\hline $\begin{array}{l}\text { Auszubildende und } \\
\text { Studierende }\end{array}$ & 10 \\
\hline
\end{tabular}

148 ff.) sozialer Netzwerke und subjektiver Wahrnehmungen von Menschen in relativer Einkommensarmut in ${ }^{5}$.

\subsubsection{Interviewleitfaden}

Die Befragten wurden gebeten, sich ca. zwei Stunden Zeit für ein Gespräch über ihr Leben und ihren Alltag zu nehmen. Um die Vergleichbarkeit der von mehreren Interviewer*innen erhobenen Daten zu gewährleisten, wurde ein Leitfaden erstellt und die Vorgehensweise in Anlehnung an das problemzentrierte Interview nach Witzel und Reiter (2012) auf mehreren gemeinsamen Workshops zu Beginn der Erhebungsphase ausführlich besprochen. Nach einer allgemeinen Interviewschulung führten alle beteiligten Interviewer*innen ein Probeinterview durch, welches dann auf einem gemeinsamen Workshop aller an der Erhebung beteiligten Personen reflektiert wurde. Dieser Workshop diente der Verbesserung der Erhebungsinstrumente und der Optimierung und Vereinheitlichung der Vorgehensweise der Interviewer*innen.

\footnotetext{
${ }^{5}$ Das Studiendesign ließ es leider nicht zu, die Datenerhebung und -analyse so miteinander zu verzahnen, dass wir von der Analyse zurück in die Datenerhebung gehen konnten, um im Feld noch einmal gezielt nach Kontrasten zu den bereits erhobenen Fällen zu suchen (Ausnahme: Nacherhebung der Interviews mit acht einkommensarmen Studierenden). Doch auch der verwendete Kompromiss einer möglichst breiten Streuung zuvor festgelegter kategorialer Eigenschaften der Befragten führte dazu, dass im Sample unterscheidbare Wahrnehmungsund Bewältigungsweisen zu finden sind und die theoretische Sättigung der Typologie erreicht werden konnte.
} 
Grundsätzlich wurden die Interviewer*innen angehalten, den Erzählfluss der Befragten anzuregen und nicht zu unterbrechen. Die Befragten sollten sich ermuntert fühlen, eigene Schwerpunkte in ihrer Erzählung zu wählen. In der Regel folgten die Interviews daher der inneren Logik der Erzählung der Befragten und nicht der Reihenfolge der im Leitfaden aufgezählten Themenschwerpunkte. Nur wenn die Befragten ein bestimmtes Thema nicht von sich aus ansprachen, sollten die Interviewer*innen auf die im Leitfaden formulierten Fragestellungen zurückgreifen. Häufig bot bereits die Antwort auf die Einstiegsfrage genügend Anknüpfungspunkte, um daran Nachfragen bezüglich aller interessierenden Aspekte anzuschließen. Die Interviews wurden wie folgt eingeleitet:

„Da wir uns noch gar nicht kennen und ich nichts über Sie weiß, würde ich vorschlagen, Sie erzählen mir doch einfach einmal, wie Ihr Alltag so aussieht. Vielleicht sagen Sie mir einfach, was Sie in der letzten Woche so alles gemacht haben? "Interviewleitfaden, S. 2

Folgende Themenschwerpunkte waren im Anschluss daran im Leitfaden vorgesehen:

- Freizeit und Hobby: Hier wurde nach Orten gefragt, die die Befragten in ihrer Freizeit aufsuchen, nach den Tätigkeiten, die sie dort ausüben, sowie nach den Personen, mit denen sie ihre Freizeit verbringen.

- Mobilität und Wohnraum: Insbesondere wurden die Befragten gebeten, ihre Wohnsituation zu beschreiben, ihr Wohnumfeld und ihre alltäglichen Wege.

- Konsum und Finanzen: Gefragt wurde, wie und wo die Interviewten einkaufen und welche Rolle das Geld dabei spielt. Anschließend wurden sie gebeten, ihre finanzielle Situation zu beschreiben. Am Ende dieses Teils sollte das Thema Armut erstmalig durch die Interviewer*innen erwähnt werden. Folgende Frage dazu wurde in jedem Interview gestellt: „Würden Sie sich als arm bezeichnen?"

- Ausbildung und Erwerbsbiographie: Die Befragten wurden gebeten, ihren Werdegang seit dem Schulabschluss zu erläutern.

- Gesundheit: Die Befragten wurden aufgefordert zu erzählen, wie es ihnen gesundheitlich geht.

- Unterstützung: Die Interviewten wurden gefragt, ob und wenn ja, in welchen Bereichen ihres Lebens sie schon einmal Unterstützung in Anspruch genommen haben. 
Der Leitfaden stellte sicher, dass diese Themenbereiche in jedem Interview angesprochen wurden. Zudem finden sich in fast jedem Interview Erzählungen, die mehr oder weniger weit über die im Leitfaden angesprochenen Themen hinausgehen.

\subsubsection{Quantitative Netzwerkabfrage}

Im zweiten Teil des Interviews wurden die Befragten gebeten, Angaben zu ihrem sozialen Umfeld zu machen, die die Interviewer*innen dann an einem eigens dafür mitgebrachten Laptop in die Eingabemaske des in der Software VennMaker (Gamper et al., 2012) programmierten Fragebogens eintrugen ${ }^{6}$. Folgende Personengeneratoren kamen dabei zur Anwendung:

1. Mit wem teilen Sie persönliche Gedanken und Gefühle oder sprechen über Dinge, die Sie nicht jedem erzählen würden?

2. Mit wem treffen Sie sich oder unternehmen Sie öfter etwas gemeinsam - z. B. spazieren gehen, essen oder ins Kino gehen, Sport treiben oder ausgehen?

3. Werden Sie von Personen, sozialen Gruppen, Organisationen oder Ämtern in irgendeiner Form unterstützt? Können Sie mir die Namen der Personen oder Institutionen nennen?

4. Wenn Sie dringend Geld bräuchten, sagen wir 50 Euro, wen würden Sie darum bitten, Ihnen Geld zu leihen? ${ }^{7}$

5. Und wenn Sie jemand bitten würde, ihm oder ihr Geld zu leihen, wem würden Sie 50 Euro leihen? ${ }^{8}$

6. Mit wem haben Sie gelegentlich Streit oder Konflikte?

Die Namen der genannten Personen wurden notiert und gespeichert. Von allen Alteri wurden das Geschlecht und die Art der Beziehung erhoben. Von

\footnotetext{
${ }^{6}$ Neben dieser Methode gibt es weitere Verfahren der visuellen Erhebung von Netzwerkdaten. Hollstein et al. (2020) (S. 4-6) unterscheiden vier Arten der visuellen Netzwerkerhebung: 1.) unstrukturierte Netzwerkkarten, 2.) strukturierte und standardisierte Netzwerkkarten, 3.) strukturierte, aber nich standardisierte Netzwerkkarten und 4.) teilstandardisierte Netzwerkkarten. Die hier zur Anwendung gebrachte Methode entspricht der zweiten Kategorie.

${ }^{7}$ Nach den ersten Probeinterviews entschieden wir uns, diese Frage anzupassen, denn $50 €$ erschienen den Befragten als ein sehr großer Betrag, den sie weder leihen noch verleihen würden. Als wir nach 5 bis $10 €$ fragten, wurden auf diese Frage hin mehr Personen genannt. ${ }^{8}$ siehe Fußnote 6.
} 


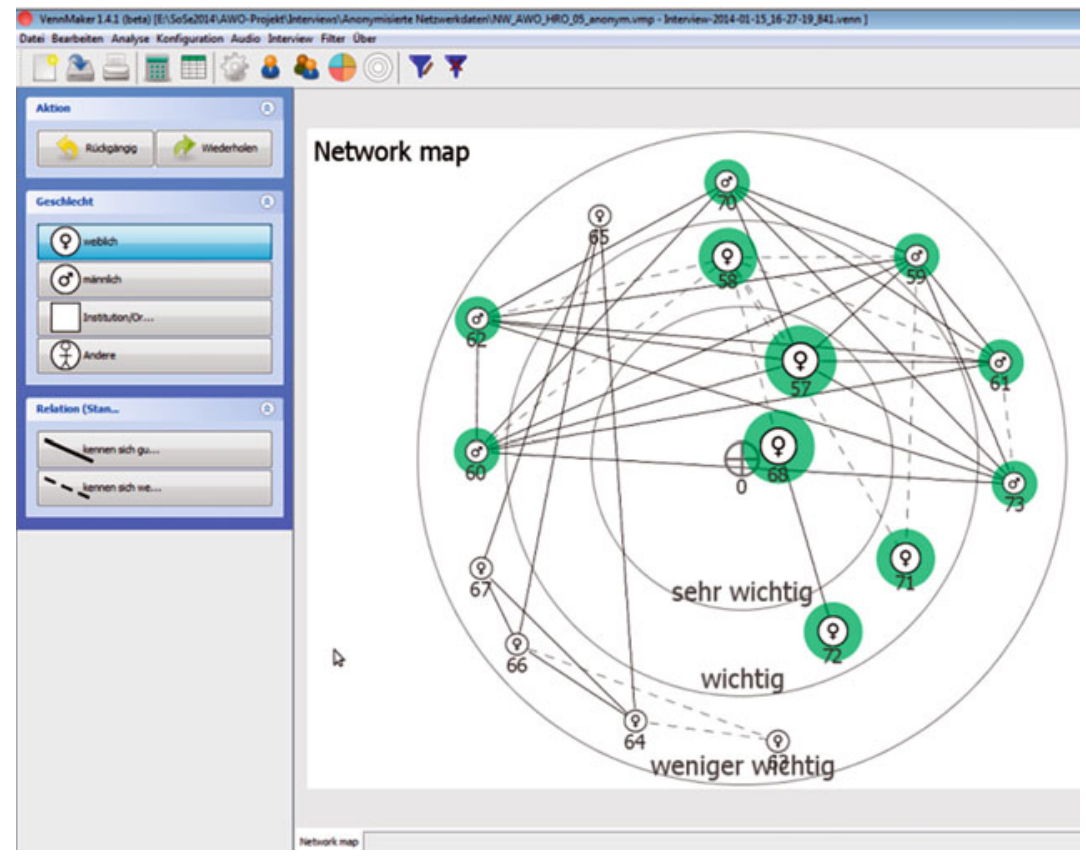

Abbildung 4.1 Netzwerkkarte in VennMaker. (Anonymisierte Darstellung)

denjenigen, die die Befragten als die 10 wichtigsten Personen im Netzwerk kategorisierten, wurden darüber hinaus folgende Eigenschaften erhoben:

- Alter

- aktuelle Erwerbstätigkeit

- Partnerschaftsform

- Kinderzahl und Alter des jüngsten Kindes

- Wohnort

- Kontakthäufigkeit zwischen Ego und Alter

- Beziehungsintensität (Wie eng fühlen Sie sich mit dieser Person verbunden?)

- Wohnentfernung zwischen Ego und Alter

Anschließend wurden die Befragten gebeten, alle genannten Personen per drag and drop auf einer Karte auf dem Bildschirm des Laptops zu platzieren, in deren Mitte sie sich selbst befinden (siehe Abbildung 4.1). Im letzten Schritt 
wurden die Alter-Alter-Beziehungen abgefragt, indem die Befragten gebeten wurden, Linien zwischen den Alteri zu ziehen, die miteinander bekannt sind. Dabei wurde zwischen starken und schwachen Beziehungen differenziert (gestrichelte Linie: kennen sich weniger gut / durchgezogene Linie: kennen sich gut).

\subsubsection{Datenbogen}

Am Ende des Interviews wurden die Befragten gebeten, einen kurzen Datenbogen mit soziodemographischen Angaben zu ihrer eigenen Person auszufüllen. Dieser enthielt neben den grundlegen soziodemographischen Indikatoren standardisierte Fragen zu Ausbildung, Erwerbsstatus, Einkommen, Haushaltszusammensetzung und Gesundheitszustand.

\subsection{Datenanalyse}

Die Arbeit an und mit den Daten vollzog sich über einen langen Zeitraum und sehr viele verschiedene Phasen hinweg, in denen immer wieder wechselnde Forschungsteams mit dem Material in Berührung kamen. Ein grober Überblick über die Arbeiten am und mit dem Material findet sich in Tabelle 4.3.

Durch diese wiederkehrende und sehr intensive Beschäftigung mit den Daten war es möglich, immer wieder neue Erkenntnisse aus unterschiedlichen Perspektiven zu gewinnen. Für die Analyseprozesse bedeutete das jedoch häufig einen Neuanfang. So wurden Teile des Materials nach der ersten eher deskriptiven Auswertung im Projektbericht hinsichtlich spezifischer Fragestellungen immer wieder unter verschiedenen Gesichtspunkten neu codiert ausgewertet. Um nicht immer wieder bei Null anfangen zu müssen, kam die Frage auf, wie bisherige Analyseergebnisse in darauffolgende Forschungen einfließen können. Dafür erwiesen sich einige Instrumente als geeigneter als andere. Codebäume (siehe unten: „Codierung") ließen sich nur sehr schwer von einer Fragestellung auf die andere übertragen, da sie einerseits sehr eng mit der Persönlichkeit der Person verbunden sind, die sie erstellt hat, und andererseits bei Veränderung der Fragestellung die Aussagekraft des Materials nicht mehr präzise genug abbilden. Hier musste von Projekt zu Projekt immer wieder neu codiert werden.

Ein zunehmend wertvolleres Analyseinstrument war dagegen die Erstellung und schrittweise Systematisierung von Fallporträts. Die anfänglich noch sehr frei als Fallmemos erstellten fallbezogenen Notizen wurden in jeder einzelnen Analyse weitergeführt. Irgendwann waren sie so umfangreich, dass sie systematisiert 
Tabelle 4.3 Bisherige Analysen unter Nutzung der in dieser Arbeit verwendeten Daten

\begin{tabular}{|c|c|c|}
\hline Zeit & Phase & beteiligte Personen \\
\hline ab Mitte 2012 & $\begin{array}{l}\text { Konzeption der Studie: Festlegung } \\
\text { der Problem- und Fragestellung in } \\
\text { Auseinandersetzung mit der } \\
\text { Auftraggeberin und Entwurf eines } \\
\text { Forschungsprogramms }\end{array}$ & $\begin{array}{l}\text { Peter A. Berger, Andreas Klärner, } \\
\text { Andreas Willisch, } \\
\text { Geschäftsführung und Vorstand } \\
\text { der AWO M-V }\end{array}$ \\
\hline \multirow[t]{3}{*}{ 03/2013-07/2014 } & $\begin{array}{l}\text { Praktische Umsetzung der } \\
\text { Erhebung }\end{array}$ & $\begin{array}{l}\text { Leitung: Andreas Klärner; } \\
\text { Rekrutierung, Organisation: } \\
\text { Marie Carnein; Durchführung: } \\
\text { Hagen Fischer, André Knabe, } \\
\text { Rainer Land, Max Leckert, Katja } \\
\text { Prochatzki }\end{array}$ \\
\hline & Transkription & $\begin{array}{l}\text { Aufträge an externe } \\
\text { Dienstleister*innen }\end{array}$ \\
\hline & $\begin{array}{l}\text { Codierung, Erstellen erster } \\
\text { Fallporträts, Diskussion der } \\
\text { Ergebnisse }\end{array}$ & $\begin{array}{l}\text { Hagen Fischer, Andreas Klärner, } \\
\text { André Knabe, Rainer Land }\end{array}$ \\
\hline $10 / 2014-12 / 2014$ & $\begin{array}{l}\text { Nacherhebung von } 8 \text { Interviews } \\
\text { mit Studierenden }+ \text { Erstellung der } \\
\text { dazugehörigen Fallporträts }\end{array}$ & $\begin{array}{l}\text { Leitung: André Knabe; } \\
\text { Praktikant*innen aus dem MA } \\
\text { Bildungswissenschaften (Uni } \\
\text { Rostock): Johannes Alisch, Pia } \\
\text { Ellgoth, Michael Könitz, Stefanie } \\
\text { Frenzel }\end{array}$ \\
\hline $09 / 2015$ & $\begin{array}{l}\text { Publikation des Projektberichts } \\
\text { (Klärner et al., 2015) }\end{array}$ & $\begin{array}{l}\text { Andreas Klärner, André Knabe, } \\
\text { Rainer Land, Peter A. Berger }\end{array}$ \\
\hline 09/2014-07/2016 & $\begin{array}{l}\text { Analysen zu Netzwerken von } \\
\text { Arbeitslosen (Klärner \& Knabe, } \\
\text { 2016) }\end{array}$ & Andreas Klärner, André Knabe \\
\hline $10 / 2015-09 / 2017$ & $\begin{array}{l}\text { Analysen zu Stigmatisierung } \\
\text { (Knabe, Fischer et al., 2018) }\end{array}$ & $\begin{array}{l}\text { André Knabe, Hagen Fischer, } \\
\text { Andreas Klärner }\end{array}$ \\
\hline $10 / 2016-04 / 2018$ & $\begin{array}{l}\text { Analysen zu negativen Aspekten } \\
\text { sozialer Beziehungen } \\
\text { (Präsentation auf der EUSN 2017) }\end{array}$ & $\begin{array}{l}\text { Philip Adebahr, André Knabe, } \\
\text { Sylvia Keim, Andreas Klärner }\end{array}$ \\
\hline 04/2016-07/2018 & $\begin{array}{l}\text { Analysen zu (Nicht-)Anerkennung } \\
\text { (Knabe, Brandt et al., 2018) }\end{array}$ & $\begin{array}{l}\text { André Knabe, Stefan Brandt, } \\
\text { Hagen Fischer, Petra Böhnke, } \\
\text { Andreas Klärner }\end{array}$ \\
\hline
\end{tabular}


Tabelle 4.3 (Fortsetzung)

\begin{tabular}{l|l|l}
\hline Zeit & Phase & beteiligte Personen \\
\hline $10 / 2016-03 / 2017$ & $\begin{array}{l}\text { Forschungspraktikum } \\
\text { „Bewältigung von Armut I“ } \\
\text { (Knabe, Aretz et al., 2018) }\end{array}$ & $\begin{array}{l}\text { Leitung: André Knabe; } \\
\text { Studierende Universität Rostock }\end{array}$ \\
\hline $10 / 2016-03 / 2017$ & $\begin{array}{l}\text { Forschungspraktikum } \\
\text { „Bewältigung von Armut II“ }\end{array}$ & $\begin{array}{l}\text { Leitung: Andreas Klärner; } \\
\text { Studierende Universität Hamburg }\end{array}$ \\
\hline $10 / 2017-07 / 2019$ & $\begin{array}{l}\text { Analysen zu Stadt-Land } \\
\text { Unterschieden (Klärner \& Knabe, } \\
\text { 2019) }\end{array}$ & Andreas Klärner, André Knabe \\
\hline
\end{tabular}

und mithilfe von Studierenden, die im Rahmen von Praktika und Forschungsseminaren mit anonymisierten Interviewtranskripten arbeiteten, zu einheitlich strukturierten Texten ausformuliert wurden. Auf diese Weise konnten die fallspezifischen Erkenntnisse von einem Analyseprozess zum nächsten bewahrt werden. Im Folgenden werden die für diese Analyse bedeutsamen Schritte im Einzelnen dargestellt.

\subsubsection{Datenaufbereitung}

Die Audiomitschnitte der qualitativen Interviews wurden wortwörtlich transkribiert und unter Verwendung der Software MaxQDA analysiert. Die mit der Software VennMaker erhobenen Netzwerkdaten wurden mit der Software R unter Verwendung der Pakete network (Butts, 2015), sna (Butts, 2016) und igraph (Csardi \& Nepusz, 2006) aufbereitet und analysiert. Grundlage der quantitativen Analyse in $\mathrm{R}$ bilden die folgenden drei Datenquellen:

1. Ein Datensatz aus 57 Netzwerkdateien, die eine Liste der Namen aller genannten Alteri beinhaltet sowie eine Edgelist, in der alle möglichen Beziehungen zwischen diesen Alteri aufgelistet sind, ergänzt um die Information, welche dieser Beziehungen tatsächlich existieren und wie stark diese Beziehung ist (0: keine Beziehung, 1: kennen sich weniger gut, 2: kennen sich gut). Diese Netzwerkdateien bilden die Grundlage für die Berechnung von Netzwerkmaßen wie Dichte und Größe sowie für die Visualisierung von Netzwerken als Soziogramm aus Knoten (Alteri) und Kanten (Beziehungen zwischen den Alteri). 
2. 57 Alteridatensätze, die die Eigenschaften der Alteri und die Informationen über die Beziehung zwischen Alter und Ego (z. B. Art, Dauer und Intensität der Beziehung) enthalten. Unter Rückgriff auf diese Daten lassen sich z. B. Analysen zur Netzwerkzusammensetzung erstellen und die zu den Alteri erhobenen Eigenschaften in den Netzwerkvisualisierungen abbilden.

3. Der Egodatensatz enthält die mithilfe des Datenbogens standardisiert erhobenen Angaben der Interviewten zu ihrer eigenen Person. Diese Informationen ermöglichen z. B. die Berechnung der Ego-Alter-Heterogenität bezüglich des Erwerbsstatus oder die Überprüfung von Korrelationen zwischen Netzwerkstrukturen und den Eigenschaften von Ego.

\subsubsection{Standardisierte Netzwerkanalyse}

Die Vorgehensweise bei der standardisierten Netzwerkanalyse unter Verwendung der drei oben beschriebenen Datensätze gliedert sich in vier Teile:

1. Visuelle Netzwerkanalyse: Für eine erste Annäherung an die Netzwerkdaten wurden zahlreiche Visualisierungen erstellt, die die erhobenen Eigenschaften der Alteri im Netzwerk abbilden. So konnten z. B. anhand der Betrachtung der Netzwerkabbildungen Thesen zur Strukturierung der Verteilung von Knoten und Kanten aufgestellt werden, z. B., inwieweit es zur Bildung von Teilgruppen von Alteri in den Netzwerken kommt und nach welchen Mustern sich diese herausbilden. Die Visualisierungen und die visuelle Analyse aller Netzwerke sind jeweils am Ende der Typenbeschreibungen in den Abschnitten 5.2.1 bis 5.2.4 zu finden.

2. Deskriptive Analyse: Um einen Überblick über das Material zu erhalten, wurden Maße zur Beschreibung der Netzwerkstruktur und -zusammensetzung für jedes einzelne Netzwerk berechnet und in einem Datensatz zusammengefasst (siehe Abschnitt 5.1.1). Dazu zählen: Netzwerkgröße, Netzwerkdichte, Modularität, Anteil familiärer Beziehungen, Anteil institutioneller Beziehungen, Anteil unterstützender Beziehungen sowie die Ego-Alter Heterogenität bezüglich des Erwerbsstatus (EI-Index).

3. Explorative Faktorenanalyse: Im nächsten Schritt wurde die Möglichkeit der quantitativen Reduktion der sieben Netzwerkvariablen auf Hauptkomponenten überprüft (auf Basis der Pearson-Korrelationsmatrix mit dem principal-Befehl aus demem psych-Paket in R, Revelle, 2017). Eine detaillierte Beschreibung der Vorgehensweise findet sich in Abschnitt 5.1.3. 
4. Clusteranalyse: Die sieben Variablen wurden schließlich einer Clusteranalyse unterzogen, die unterschiedliche Netzwerkstrukturen und -zusammensetzungen im Sample identifiziert. Die Clusteranalyse wurde unter Anwendung der „Hierarchical Clustering“-Funktion (hclust) des Stats-Pakets in R durchgeführt (R Core Team, 2017). Zur Anwendung kam Ward's minimum variance method (,ward.d2“, vgl. Ward, 1963), die im Vergleich zu anderen Verfahren die treffendsten Cluster liefert. Die Vorgehensweise wird im Abschnitt 5.1.4 Schritt für Schritt erläutert und begründet.

\subsubsection{Codierung}

Bei einer ersten Sichtung des qualitativen Materials wurden Kategorien in Anlehnung an die Vorgehensweise der Grounded Theory (Glaser \& Strauss, 1967; Strauss \& Corbin, 1990) gebildet. Dazu wurden Textpassagen markiert und mit Kurzbeschreibungen versehen, die beim Lesen einen besonderen Eindruck hinterlassen haben oder die Aussagen bezüglich des Erkenntnisinteresses dieser Arbeit enthalten (offenes Codieren, nach Boehm, 1994, 127 ff.; Strauss \& Corbin, 1996, 43 ff.). In einem zweiten Schritt wurde diese lose Sammlung von Open Codings zu Kategorien verdichtet, in denen thematisch ähnliche Codings zusammengeführt wurden (axiales Codieren nach: Boehm, 1994, 130 ff. und Strauss \& Corbin, 1996, 75 ff.). Parallel zu dem fallübergreifenden Codesystem wurden fallspezifische Memos und Diagramme erstellt (in Anlehnung an: Strauss \& Corbin, 1996, 169 ff.), in denen die Beobachtungen der beteiligten Forscher*innen zunächst unstrukturiert festgehalten wurden, um dann im Rahmen eines diskursiven Prozesses immer wieder überarbeitet und schrittweise systematisiert zu werden. Aus dieser Prozedur entsprangen erste Theorien darüber, auf welche Weise sich die Wahrnehmungs- und Bewältigungsweisen der Befragten ähneln oder unterscheiden (Prinzip der komparativen Analyse nach Glaser \& Strauss, 2010, 32 ff.).

\subsubsection{Fallporträts}

Als Grundlage für die Bildung einer Typologie musste die Analyseperspektive von der Gesamtbetrachtung des Samples auf die Einzelfälle gelenkt werden. Hierzu wurden die im Codierprozess erarbeiteten Kernkategorien sowie die bis hierhin erstellten Fallmemos wie folgt zu Gliederungspunkten vorstrukturierter Fallporträts weiterentwickelt (siehe auch: Knabe, Aretz et al., 2018, 12 f.): 
- Deckblatt: Jedes Porträt erhielt ein Decklblatt auf dem die wichtigsten soziodemographischen Angaben sowie einige Stichpunkte zu den im Folgenden ausgeführten Kategorien festgehalten wurden. Das Deckblatt diente dazu, die im Porträt enthaltenen Informationen zum Fall im Rahmen der Gruppenarbeiten an der Typologie schnell abrufen zu können.

- Einleitende Beschreibung der Person: Hier wurden besonders charakteristische Eigenschaften und Aussagen der Person festgehalten.

- Biographie: Die wichtigsten Lebensereignisse der Interviewten wurden chronologisch festgehalten und eine Skizze über die ,biographische Gesamtformung, d. h., die lebensgeschichtliche Abfolge der erfahrungsdominanten Prozeßstrukturen in den einzelnen Lebensabschnitten bis hin zur gegenwärtig dominanten Prozeßstuktur“ (Schütze, 1983, zitiert nach: Flick, 2016, S. 437) erstellt.

- Aktuelle Lebenssituation: Thema dieses Abschnitts war die Beschreibung der Alltagsstruktur der Befragten und der darin verübten Tätigkeiten. Darüber hinaus sollten die Wohnsituation sowie die sozialen und räumlichen Kontexte der Alltagspraktiken der Befragten umrissen werden.

- Soziale Einbindung: Hier ging es darum, die Beziehungen zwischen den Akteur*innen innerhalb der sozial-räumlichen Kontexte zu beschreiben. Zunächst sollten alle alltagsrelevanten Personen und Institutionen notiert werden, die im Interview genannt wurden. Dann sollte in Anlehnung an die Qualitativ Strukturale Analyse (Herz et al., 2015) erstens zusammengefasst werden, in welcher Beziehung die jeweiligen Akteur*innen zu Ego stehen, und zweitens, wie sich die Akteur*innen im Netzwerk wechselseitig zueinander verhalten.

- Materielle Situation: Festgehalten wurden die Konsumstrategien der Befragten sowie die subjektive Einschätzung ihrer finanziellen Lage.

- Perspektiven: Schließlich wurde festgehalten, welche Ziele die befragte Person im Hinblick auf ihre Zukunft benennt. In Anlehnung an die wissenssoziologische Perspektive (P. L. Berger \& Luckmann, 2013) sollte die „Welt in potentieller Reichweite“ (Schütz \& Luckmann, 2003, S. 72) anhand der folgenden vier Dimensionen skizziert werden (ebd. S. 73 ff.): Biographische Erfahrungen (Welche Herausforderungen wurden in der Vergangenheit bewältigt?), Wahrnehmung räumlicher Distanzen zu möglichen Handlungszielen, Wahrnehmung verfügbarer Zeithorizonte und -budgets, Wahrnehmung der eigenen sozialen Position und ihrer Entwicklungspotentiale.

$\mathrm{Zu}$ jedem dieser Punkte sollten aussagekräftige Zitate der Interviewten in das Porträt eingefügt werden. 
Ein Teil der etwa zehnseitigen Fallporträts wurde bereits vom Projektteam aus der Studie „Aspekte der Armut in Mecklenburg-Vorpommern“ unter Mitwirkung des Autors dieser Arbeit erstellt (Klärner et al., 2015). Weitere Porträts entstanden unter Anleitung des Autors im Rahmen eines Forschungspraktikums mit Studierenden der Universität Rostock im Wintersemester 2016/' 17 (Knabe, Aretz et al., 2018) sowie im Rahmen eines zeitgleich durchgeführten Forschungsseminars an der Universität Hamburg unter Leitung von Andreas Klärner. Die Porträts zu den im Rahmen der Nacherhebung durchgeführten acht Interviews mit Studierenden wurden von den Interviewer*innen selbst verfasst (siehe Tabelle 4.3).

\subsubsection{Typisierung}

Die Typologie wurde parallel zum bis hierhin beschriebenen Analyseprozess erstellt und darüber hinaus weiterentwickelt. Erste Ideen für Kategorien zur Unterscheidung von Handlungs- und Bewältigungsstrategien entstanden im Rahmen der Codierung (siehe oben). Im Sinne des Theoretical Samplings aus der Grounded Theory (Glaser \& Strauss, 2010, 53 ff.; Strauss \& Corbin, 1996, 148 ff.) wurde nach möglichst kontrastiven Wahrnehmungs- und Bewältigungsweisen innerhalb des Samples gesucht ${ }^{9}$. Die gefundenen Gegensätze dienten der Formulierung von Kategorien und Dimensionen der Unterscheidung von Fällen. So konnten z. B. entlang der Dimension „Selbstwirksamkeit“ Unterschiede zwischen solchen Interviewten festgestellt werden, die ihr eigenes Handeln als eher fremdbestimmt und solchen, die ihre Handlungen als eher selbstbestimmt wahrnehmen. Die gefundenen Kategorien wurden über den gesamten Analyseprozess hinweg immer wieder mit Kolleg*innen und Studierenden diskutiert und weiterentwickelt (siehe Tabelle 4.3 auf S. 96).

Einer der ersten Schritte in Richtung der in dieser Arbeit finalisierten Typisierung war die intensive Diskussion der Fallporträts mit den Teilnehmenden des oben bereits erwähnten Forschungspraktikums im Wintersemester 2016/' 17 an der Universität Rostock (Knabe, Aretz et al., 2018). Hier wurden 14 der 57 Fälle in acht anderthalb- bis zweistündigen Diskussionen besprochen, miteinander verglichen (ebd. S. 12 ff.) und zu einer Typologie dieses Teilsamples verdichtet (ebd. S. 17). Die Vorgehensweise hierbei kann als idealtypisch für die Arbeit an der in dieser Arbeit finalisierten Typologie aller 57 Fälle beschrieben werden und soll daher kurz zusammengefasst werden.

\footnotetext{
${ }^{9}$ siehe Fußnote 4.
} 
- Methodische Grundlage: Die Ausarbeitung der Typologie orientierte sich an der Typisierung im Rahmen der dokumentarischen Methode, bei der zunächst typische Deutungs- und Orientierungsmuster handelnder Subjekte rekonstruiert werden sollen (Motive, Mittel und Strategien von Handlungen), um darauf aufbauend zu erklären, auf welche Weise diese Muster zustande kommen (Bohnsack, 2013): Demnach werden Typologien in zwei Schritten erstellt, die als sinngenetische und soziogenetische Typisierung bezeichnet werden. Ziel der sinngenetischen Typisierung ist die Identifikation von Fällen mit ähnlichen Orientierungsrahmen. Hier werden Fälle zu Typen verdichtet, die sich hinsichtlich der Wertvorstellungen und Handlungsweisen der Befragten gleichen (Bohnsack, 2013). Bei der soziogenetischen Typisierung geht es dann darum, die sozialen und materiellen Ursprünge dieser Orientierungsrahmen zu identifizieren, bzw. „die Genese des jeweiligen Orientierungsrahmens“ (Nohl, 2013, S. 38) zu rekonstruieren.

- Sinngenetische Typisierung: Grundlage der sinngenetischen Typisierung waren die Fallporträts. Diese wurden in einem sukzessiven Prozess miteinander verglichen. Für die Erstellung der ersten Typologie mit 14 Fällen im Seminarkontext wurden jedem bzw. jeder Teilnehmenden zwei Fälle zugewiesen, zu denen Fallporträts erstellt und vor der Gruppe präsentiert werden sollten. Nach der Präsentation jedes Falls hatten die übrigen Teilnehmenden die Gelegenheit, Ähnlichkeiten und Unterschiede zu ihren eigenen Fällen zur Diskussion zu stellen. Das Diskussionsergebnis wurde durch den Seminarleiter visualisiert, der die Deckblätter der Fallporträts nach den Anweisungen der Seminarteilnehmenden auf einer Tafel anordnete. Sinngenetisch ähnliche Fälle wurden nah beieinander platziert, während Unterschiede durch größere Distanzen zwischen den Deckblättern symbolisiert wurden. Mit jedem neu hinzukommenden Fall wurde die Anordnung aller bis dahin an die Tafel gehefteten Fälle wieder so lange überarbeitet, bis alle Mitglieder der Forschungsgruppe einen Konsens über die passende Positionierung jedes Falls erarbeitet hatten. Ganz im Sinne der Weber'schen Überlegungen zum „Idealtypus“ (Weber, 1988, 190 f.) verlagerte sich der Fokus dieser Diskussionen im Laufe des Prozesses von den Besonderheiten einzelner Fälle auf die Formulierung und Auswahl geeigneter Kategorien zu ihrer grundsätzlichen Unterscheidung. In den so entstandenen Fallgruppen wurden häufig auftauchende Muster zu regelmäßigen Handlungsweisen innerhalb eines Idealtyps 


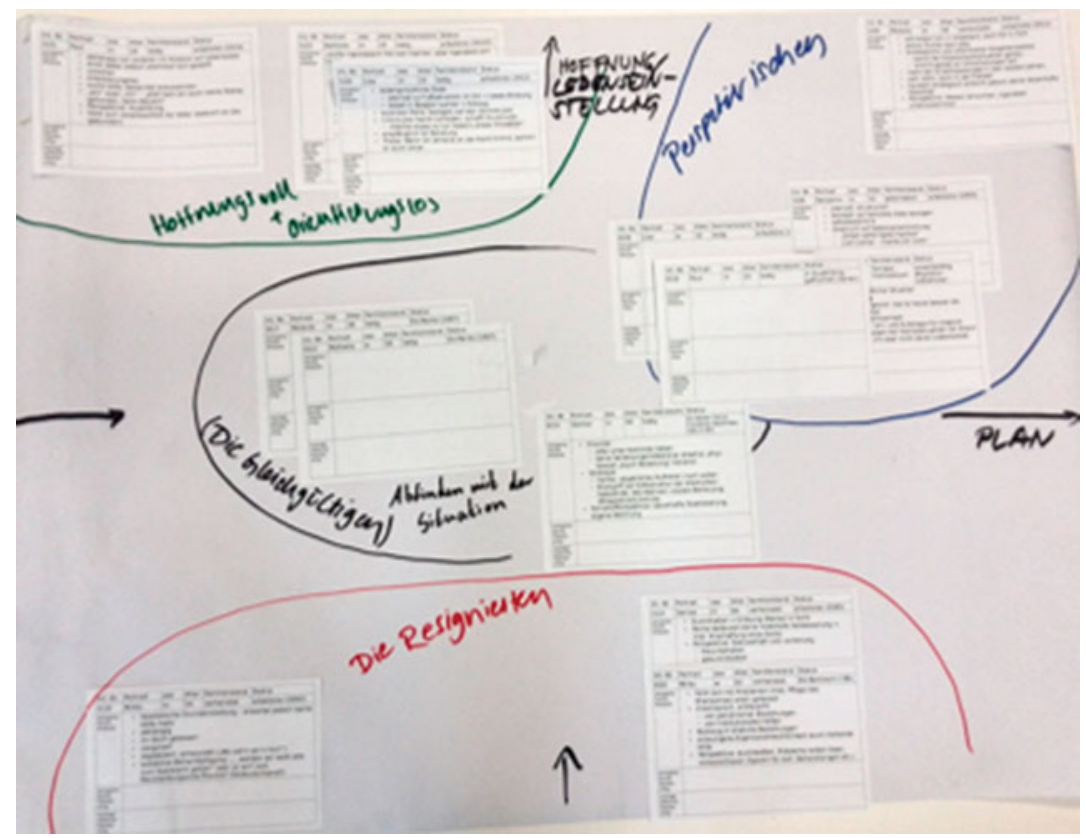

Abbildung 4.2 Typologie des Seminars am Beginn des Diskussionsprozesses

verdichtet, während fallspezifische Besonderheiten mehr und mehr in den Hintergrund traten ${ }^{10}$.

- Soziogenetische Typisierung: Im zweiten Schritt wurde nach soziogenetischen Gemeinsamkeiten innerhalb der sinngenetischen Typen gesucht bzw. nach entsprechenden Unterschieden zwischen den Typen (,,interne Homogenität“/,externe Heterogenität“" nach Kelle \& Kluge, 2010, S. 85). Die gefundenen Kriterien wurden zunächst durch Pfeile und Notizen auf der Schautafel visualisiert und sukzessive zu Diemensionen in einem Merkmalsraum verdichtet. In

\footnotetext{
${ }^{10}$ Nach Weber wird der Idealtypus , gewonnen durch einseitige Steigerung eines oder einiger Gesichtspunkte und durch Zusammenschluß einer Fülle von diffus und diskret, hier mehr, dort weniger, stellenweise gar nicht, vorhandenen Einzelerscheinungen, die sich jenen einseitig herausgehobenen Gesichtspunkten fügen, zu einem in sich einheitlichen Gedankenbilde. In seiner begrifflichen Reinheit ist dieses Gedankenbild nirgends in der Wirklichkeit empirisch vorfindbar [...]“ (Weber 1988, S. 191).
} 


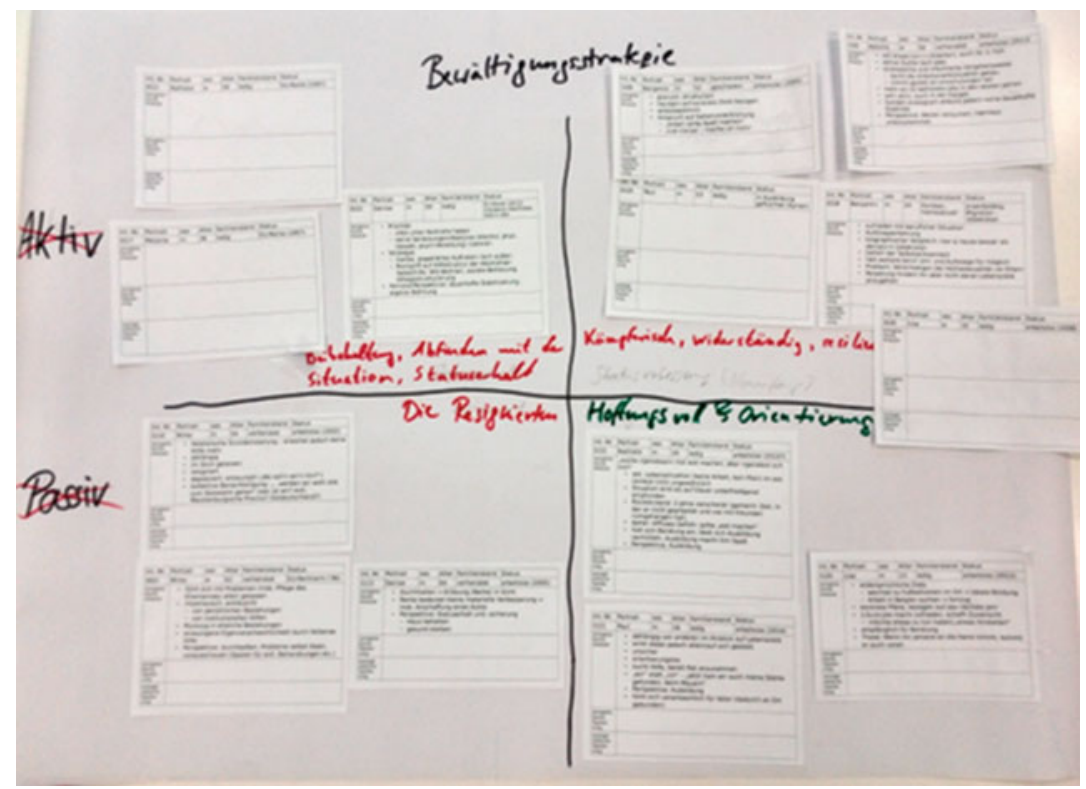

Abbildung 4.3 Typologie des Seminars zur Mitte des Diskussionsprozesses

den Abbildungen 4.2 bis 4.4 ist $\mathrm{zu}$ sehen, wie sich die Typologie im Laufe dieses Prozesses entwickelt hat.

Nach Beendigung des Forschungspraktikums und Veröffentlichung eines gemeinsamen Berichts mit den Studierenden (Knabe, Aretz et al., 2018) erfolgte die Weiterentwicklung, Überarbeitung und Erweiterung der ursprünglichen Typologie durch die schrittweise Hinzunahme aller weiteren Fälle aus dem Sample. In diesem Prozess wurden immer wieder neue Gruppen gebildet oder bereits formulierte Typen mit anderen zusammengefasst, bis Kriterien gefunden waren, nach denen sich alle Fälle sinnvoll strukturieren und typisieren ließen. Nachdem etwa 30 Fälle in die Typologie eingearbeitet waren, nahmen diese Umformulierungen der Typologie ab. Ab diesem Zeitpunkt ließen sich fast alle weiteren Fälle gut in die bestehenden Typen einordnen, ohne dass größere Änderungen nötig waren. Damit war das Kriterium der theoretischen Sättigung in der Typenbildung erreicht (siehe Fußnote 4). 


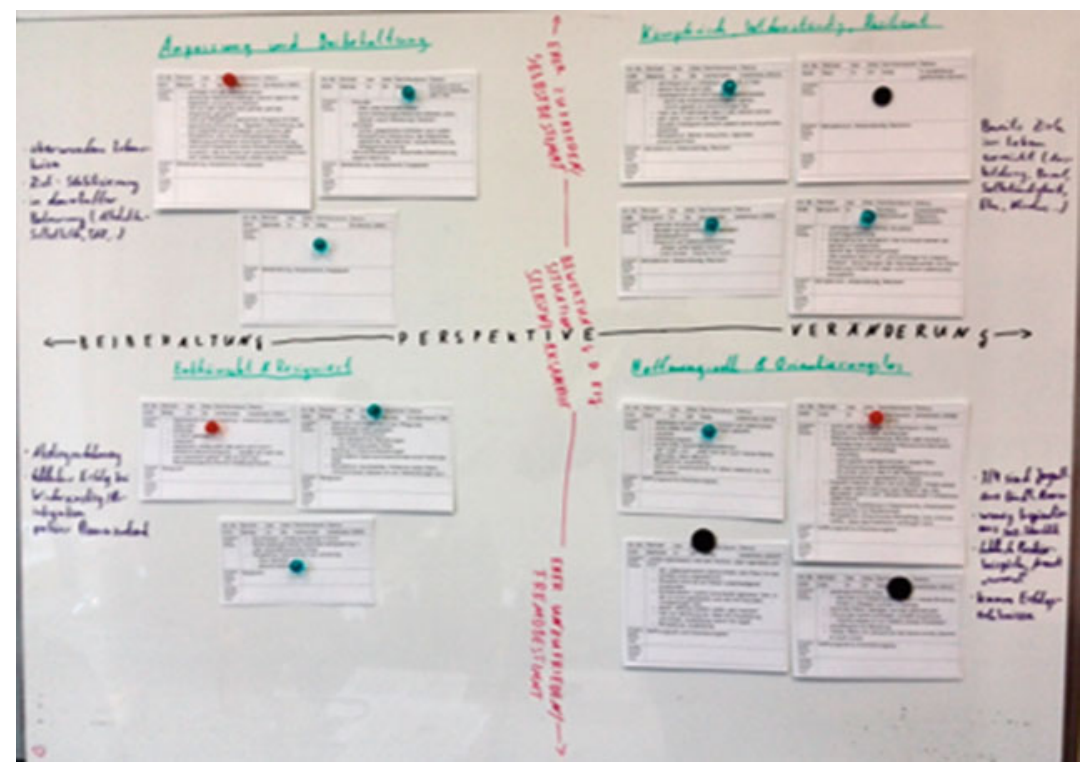

Abbildung 4.4 Typologie des Seminars gegen Ende des Diskussionsprozesses

Am Ende des Auswertungsprozesses erfolgt der Vergleich zwischen der quantitativen Analyse und der qualitativen Typologie. Die Chancen und Risiken dieser methodenintegrativen Vorgehensweise werden im folgenden Abschnitt diskutiert.

\subsection{Exkurs zum Verhältnis qualitativer und quantitativer Analysen in den Sozialwissenschaften}

In jüngster Zeit wird in der Soziologie wieder häufiger über die richtige Methode diskutiert. Symbolisch hierfür steht der Konflikt zwischen der eher auf Methodenintegration angelegten Deutschen Gesellschaft für Soziologie und der im Juli 2017 gegründeten eher am quantitativen Paradigma orientierten Akademie für Soziologie. Noch in dem im Jahr 2017 erschienenen Sonderband der Kölner Zeitschrift für Soziologie und Sozialpsychologie zu „Mixed Methods “ (Baur et al., 2017a) wurde die bis dato lange zu beobachtende „friedliche Koexistenz“ 
(Baur et al., 2017b, S. 9) zwischen dem qualitativen und dem quantitativen Paradigma in der deutschsprachigen Soziologie als schädlich für die Entwicklung der sozialwissenschaftlichen Methodik beklagt, denn:

„Aus einer methodenintegrativen Perspektive kann dies kaum ein erstrebenswerter Zustand sein, denn in einer solchen (nur scheinbar befriedeten) Situation kann jenes Potential für Methodenentwicklung und Methodenverbesserung, das aus einer kritischen und manchmal auch kontroversen Diskussion erwächst, nicht genutzt werden. " (Baur et al., 2017b, S. 9)

Die Autor*innen sahen in dieser vermeintlichen Ruhe der Auseinandersetzung das „Fundamentalprinzip von Mixed Methods“ (ebd. S. 9) bedroht, welches auf „der Einsicht [beruht], dass sowohl qualitative als auch quantitative Methoden spezifische Grenzen und Probleme aufweisen, die den Rückgriff auf Verfahren aus der jeweils anderen Tradition geradezu zwingend machen“ (ebd.). Eine Aufteilung in zwei unabhängig voneinander arbeitende Lager sei kontraproduktiv für die Entwicklung der sozialwissenschaftlichen Methodik insgesamt.

Dieser Forderung nach einer wechselseitigen Bereicherung und Kritik quantitativer und qualitativer Methoden möchte diese Arbeit nachkommen. Allerdings ergibt sich aus dieser Herangehensweise eine etwas unkonventionelle Datengrundlage und -analyse, die in Diskussionen mit Kolleg*innen immer wieder Fragen aufwirft, die es wert sind, tiefergehend betrachtet zu werden. Zwei Fragenkomplexe sind dabei von wiederkehrender Bedeutung:

1. Um welche Art von Daten handelt es sich hier eigentlich? Was daran ist qualitativ, was quantitativ? Ab wann darf von einem Mixed-MethodsDesign gesprochen werden? Und: Welcher Mehrwert ergibt sich aus dieser Perspektive im Vergleich zu rein qualitativen oder rein quantitativen Ansätzen?

2. Welche Bedeutung haben Visualisierungen sozialer Netzwerke im Rahmen der Analyse? Handelt es sich bei ihrer Analyse um eher qualitative oder um eher quantitative Verfahren? Sind sie mehr als eine exemplarische Illustration der Befunde?

Diesen Fragen sollen in den folgenden beiden Abschnitten unter Bezugnahme auf die in dieser Arbeit zur Anwendung gebrachte Methode erörtert werden. 


\title{
4.3.1 Quali, Quanti oder Mixed Methods?
}

Die in dieser Arbeit verwendeten Datenerhebungs- und Analysemethoden lassen sich ohne Zweifel als Methodentriangulative soziale Netzwerkanalyse (bzw. SNA: Social Network Analysis) bezeichnen. Es kommen standardisierte und nichtstandardisierte Instrumente zur Erfassung und Interpretation von Netzwerkdaten zum Einsatz. Ob man deshalb schon von einer Mixed-Methods-Studie sprechen sollte, ist abhängig davon, was genau darunter verstanden wird. Bedeutet Mixed Methods eine Methodentriangulation, die sich beider Paradigmen bedient und ihre Schlüsse aus der Integration qualitativer und quantitativer Verfahrensweisen zieht (Creswell 2016, S. 216 f.), kann die Frage bejaht werden. Legt man jedoch strengere Kriterien an, könnte die Bewertung, insbesondere in Bezug auf den quantitativen Teil der Analyse, anders ausfallen: Baur et al. (2017b) zufolge müssen folgende Gütekriterien erfüllt sein, um von quantitativer Forschung sprechen zu können: die Objektivität, die Intersubjektivität, die Wiederholbarkeit und die Repräsentativität der Beobachtungen (ebd., S. 9 f.). Die hier vorgenommene standardisierte Messung von Netzwerken kann durchaus als ein Versuch der Objektivierung und intersubjektiven Erfassung sozialer Strukturen sowie der Ermöglichung der Wiederholbarkeit der Messung durch andere Forscher*innen gezählt werden. Die ersten drei Kriterien wären damit erfüllt. In Sachen Repräsentativität sind der hier durchgeführten Untersuchung aufgrund der geringen Fallzahl jedoch klare Grenzen gesetzt.

Schaut man etwas tiefer in die methodologischen Diskussionen innerhalb der Netzwerkforschung, fällt auf, dass Repräsentativität hier grundsätzlich und unabhängig vom zugrundeliegenden Paradigma nur schwer herzustellen ist. So wird diskutiert, auf welcher Erklärungsebene Netzwerkstudien anzusiedeln sind und wie repräsentativ sie sind (Hollstein, 2014). Bellotti (2010) bezeichnet in diesem Zusammenhang sogar hoch standardisierte Netzwerkstudien als „micro“:

\begin{abstract}
"However, I do believe that even when quantitative tools are applied to networks, the object of analysis is often micro: in stochastic actor-based models for network dynamics, for example, "the empirical data consist of two, but preferably more, repeated observations of a social network on a given set of actors" (Snijders, van de Bunt, and Steglich 2010)[ ${ }^{11}$ ]. In these cases networks consist of an entire population: the main problem, here, is how to set the boundaries of natural networks. But once they are defined, results are valid only for those specific networks, even if they can still be useful to formulate structural hypothesis to be tested in further studies." (Bellotti, 2010, S. 5)
\end{abstract}

${ }^{11}$ Snijders, T.A.B., van de Bunt, G.G., and Steglich, C.E.G. 2010 "Introduction to Stochastic Actor-Based Models for Network Dynamics.” Social Networks 32: 44-60. 
Demnach sind die Aussagen, die auf Basis von Gesamtnetzwerkanalysen getroffen werden, zwar sehr präzise für ebenjene, doch nicht unbedingt repräsentativ in Bezug auf andere Netzwerke. Oder anders ausgedrückt: Nur weil die Prozesse in einer Schulklasse, einer ganzen Schule oder sogar einer Kleinstadt auf bestimmte Weise ablaufen, heißt das nicht, dass sich diese Erkenntnisse ohne weiteres auf andere Klassen, Schulen oder Städte übertragen lassen.

Das Problem mangelnder Repräsentativität sollte jedoch nicht dazu führen, dass man auf derartige Erhebungen verzichtet - unter Umständen will man ja gar keine allgemeingültigen Aussagen treffen, sondern die Prozesse in einem spezifischen Kontext analysieren. Wichtig ist, dass man solche methodischen Probleme zum Anlass nimmt, die eigenen Vorgehensweisen und Schlussfolgerungen zu reflektieren. Eine Möglichkeit, der fehlenden Repräsentativität aufgrund einer eher geringen Fallzahl zu begegnen, besteht in der Anpassung der Sampling Strategie. Das Quota Sampling (Przyborski \& Wohlrab-Sahr, 2014, S. 184) hält dafür geeignete Verfahrensweisen bereit (zu finden bei: Bernardi, 2011; Bernardi et al., 2014, 126 f.; Bolíbar, 2016): Dabei wird eine Fallzahl festgelegt, die mindestens erreicht werden soll, um später Berechnungen mit den erhobenen Daten durchzuführen zu können. Darüber hinaus werden Varianzen im Hinblick auf bestimmte Eigenschaften der Befragten minimiert, indem z. B. nur Angehörige einer bestimmten Einkommensgruppe in das Sample aufgenommen werden. Andere Eigenschaften werden dagegen bewusst variiert, um Analysen bezüglich der Unterschiede zwischen diesen Gruppen zu ermöglichen. Damit beschränkt sich die Aussagekraft der Daten auf eine kleinere, dafür aber präziser abgrenzbare Gruppe, für die ein gewisses $M a ß$ an Repräsentativität hergestellt werden kann.

Es sollte also nicht um die möglichst orthodoxe Anwendung einer Methode gehen, sondern darum, sich ihre spezifischen Vor- und Nachteile bewusst zu machen und Strategien im Umgang mit den Defiziten der gewählten Vorgehensweise zu entwickeln. Ausgangspunkt der Suche nach der geeigneten Methode oder dem am besten geeigneten Methodenmix sollte die Frage sein, worüber genau eine Untersuchung eigentlich Auskunft geben soll:

- Quantitative Analysen sind immer dann von Nutzen, wenn es um einen grundsätzlichen Überblick über die zu einem bestimmten Zeitpunkt gegebene Netzwerkstruktur geht (Bolíbar, 2016). Mit ihnen lassen sich Netzwerke von außen sehr gut beobachten - daher wird in diesem Zusammenhang auch vom „Outsider View“ gesprochen (Edwards, 2010; Jack, 2010).

- Mit qualitativen Methoden lässt sich mehr darüber erfahren, welche Bedeutungen auf der subjektiven Ebene mit diesen Beziehungsstrukturen verbunden, 
bzw. wie und warum diese entstanden sind (Bolíbar, 2016, S. 2227; Crossley, 2010; Crossley et al., 2015, 106 ff.).

- Durch die Variation der Perspektive auf das zu untersuchende Phänomen können Mixed Methods darüber hinaus die gewonnenen Erkenntnisse validieren oder blinde Flecke in der einen oder anderen Erhebungs- und Analysemethode identifizieren (Rice et al., 2014). So lassen sich etwa schwache Beziehungen nur schwer durch standardisierte Namensgeneratoren erfassen (Mønsted, 1995). Die eher zufälligen oder flüchtigen Begegnungen, die Potentiale für Innovationen in Netzwerken bereithalten (Strength of weak ties - Granovetter, 1973), werden häufig eher auf Nachfrage einer aufmerksamen Interviewerin hin beschrieben als in einer standardisierten Abfrage benannt (Edwards, 2010).

Mixed Methods Network Analysis fragt danach, auf welche Weise Netzwerkstrukturen und Netzwerkkulturen miteinander interagieren (Crossley et al., 2015, 106) und gibt so eine methodologische Antwort auf den bereits im Theorieteil ausbuchstabierten Cultural Turn in der Netzwerkforschung (siehe Abschnitt 2.2.3, S. 37 ff.). Analysiert werden Netzwerke nicht mehr als leere Strukturen, sondern als systematische Anordnungen von Bedeutungen. Die den Beziehungen innewohnenden Stories und die domänenspezifischen Kulturen müssen im Rahmen qualitativer Analysen rekonstruiert werden. Fuhse (2009) spricht in diesem Zusammenhang von „Bedeutungsstrukturen sozialer Netzwerke“:

„The meaning structure of social networks consists of interpersonal expectations. These are embodied in dyadic relationships and cultural blueprints for these, in role categories, and in social identities of actors." (Fuhse, 2009, 67f.)

Mixed-Methods-Studien können der Frage nach dem Zusammenhang zwischen Bedeutungs-und Netzwerkstrukturen nachgehen, indem sie beide Ebenen erfassen und analysieren (Crossley et al., 2015, 116 f.; Fuhse, 2009, S. 68). Diese Analyse kann ihren Ausgangspunkt entweder bei der standardisiert erfassbaren Netzwerkstruktur oder bei der qualitativ rekonstruierten Bedeutungsstruktur nehmen. Im ersten Fall wird gezeigt, wie Netzwerkstrukturen Bedeutungsstrukturen beeinflussen. Es wird rekonstruiert, ob und auf welche Weise die Einbettung in systematisch unterscheidbare Netzwerkstrukturen (z. B. in unterschiedliche Typen egozentrierter Netzwerke) systematisch unterschiedliche Handlungspraxen hervorbringt. Im zweiten Fall wird gezeigt, inwiefern Bedeutungsstrukturen Netzwerke formen. Es wird rekonstruiert, welche Auswirkungen netzwerkspezifische Deutungsmuster auf das Beziehungshandeln von Akteur*innen ausüben (ebd.). 
Dennoch stößt man immer wieder auf Probleme bei der Präsentation von Ergebnissen, die sich - glücklicherweise nur sehr selten auf diese hier etwas zugespitzte Weise - in folgendem Dilemma äußert: Die einen verweigern die Aufmerksamkeit für die Analysen aufgrund einer zu geringen Fallzahl, die anderen, weil sie die Graphen und Maßzahlen für irrelevant und oberflächlich halten.

Der Mixed-Methods-Begriff könnte für einen Ausweg aus diesem Dilemma sorgen, wenn er nicht als drittes Lager, sondern als Aufforderung an beide Seiten verstanden wird, die eigene Vorgehensweise durch die Einnahme einer alternativen Perspektive zu hinterfragen. Die von Hollstein (2014, S. 11) formulierten Kriterien zur Bestimmung einer Studie als Mixed Methods Social Network Analysis geben Forschenden dafür eine pragmatische Minimaldefinition an die Hand. Demnach müssen in diesen Studien:

1. qualitative und quantitative Daten verwendet werden, die sich auf Knoten und die Beziehungen dazwischen beziehen,

2. diese Relationen und Netzwerke unter Anwendung statistischer Verfahren und interpretativer Strategien analysiert werden und

3. die qualitativen und quantitativen Ansätze aufeinander bezogen werden (Integration).

Wir haben es in dieser Arbeit also mit einer Mixed Methods Social Network Analysis nach Hollstein (2014) zu tun: Es wurden quantitative und qualitative Netzwerkdaten erhoben, die unter „Anwendung statistischer Verfahren (deskriptive Statistik, Faktorenanalyse, Clusteranalyse) und interpretativer Strategien (Codierung, Typisierung) analysiert wurden und in einem dritten Schritt aufeinander bezogen werden (Vergleich der quantitativen und qualitativen Typisierung).

\subsubsection{Die Sonderrolle der Visuellen Netzwerkforschung}

Seit der Erfindung des Soziogramms (Moreno, 1934) werden visuelle Netzwerkdarstellungen in der Psychologie und den Sozialwissenschaften erhoben und analysiert. In den letzten Jahren lässt sich ein Übergang vom Soziogramm zum Netzwerkgraphen feststellen (Tubaro et al., 2016, S. 2): freie PaperPencil-Zeichnungen werden immer häufiger durch Plots standardisiert erhobener Netzwerkdaten ersetzt, die mithilfe von Software erstellt werden (Gamper et al., 2012; McCarty, 2003). Während freie Netzwerkzeichnungen noch relativ leicht dem qualitativen Paradigma zuzuordnen waren, lässt sich die visuelle Analyse 
standardisierter Netzwerkdaten weder eindeutig als qualitative noch als quantitative Methode kategorisieren. $\mathrm{Zu}$ sehr ist die Deutung dieser Daten abhängig von Entscheidungen für oder gegen eine schier unüberschaubare Anzahl an Algorithmen und Maßzahlen, die in einem oft sehr undurchsichtigen und kaum dokumentierten Prozess gefällt werden. Yousefi Nooraie et al. (2020) beschreiben diesen Vorgang in Bezug auf Netzwerk-Visualisierungen wie folgt:

"There is no single accepted approach to graphical depiction of social networks. The
researcher chooses the most satisfactory presentation through a subjective and repeti-
tive process of trying afew layout algorithms (which refer to the methods of distributing
nodes in the map), adjusting the sizes, colors, and shapes of the nodes according to
the most informative/distinctive personal attributes, adjusting the thickness and the
length of ties (to represent the strength of relations and the distance between groups),
and highlighting social clusters." (Yousefi Nooraie et al., 2020, $115 \mathrm{f}$.)

Sobald sich die Forschenden für ein bestimmtes Netzwerklayout entscheiden oder Attribute auswählen, die sie im Netzwerk darstellen möchten, laden, sie die Plots mit Bedeutungen auf, die nicht mehr nur aus den Daten selbst stammen:

"Interpretation of a network graph is not only influenced by the structure of the data
and analytical choices but is also developed in light of the background knowledge of the
observer about the social dynamics and patterns being depicted." (Yousefi Nooraie
et al., 2020, S. 116 unter Verweis auf: Blythe et al. 1995 und McGrath et al. 2014)

Sowohl die Erstellung von Visualisierungen als auch deren Interpretation kommen nicht ohne reflexive Deutungsprozesse aus, die wir eher in der Grounded Theory (z. B. bei Breuer et al., 2019) verorten würden als in der rein quantitativ arbeitenden Sozialforschung. Diese Beobachtung lässt sich sehr gut anhand der Arbeit von Bidart et al. (2018) illustrieren. Sie schlagen vor, egozentrierte Netzwerke in vier Schritten zu typisieren:

1. Die Basis der Typologie bildet die visuelle Analyse von Netzwerkplots, in der die Forscher*innen eine erste Typisierung der erhobenen Netzwerke

\footnotetext{
${ }^{12}$ Blythe, J., McGrath, C., \& Krackhardt, D. (1995). The effect of graph layout on inference from social network data. In F. J. Brandenburg (Ed.), International symposium on graph drawing (pp. 40-51). Heidelberg, Germany: Springer. / McGrath, C., Blythe, J., \& Krackhardt, D. (2014). Visualizing multiple levels and dimensions of social network properties. In W. Huang (Ed.), Handbook ofhuman centric visualization (pp. 513-525). New York, NY: Springer.
} 
vornehmen - die Autor*innen kommen in ihrem Datensatz auf 6 visuelle Netzwerktypen (siehe Abbildung 4.5a).

2. Im Anschluss daran werden quantitative Indikatoren gesucht, die diese Typen charakterisieren. Hier wird also geschaut, inwiefern sich die Ausprägungen unterschiedlicher Netzwerkmaße innerhalb der visuellen Typen ähneln, bzw. wie stark sie sich von Typ zu Typ unterscheiden.

3. Dann wird auf Basis der in Schritt 2 als geeignet identifizierten Netzwerkmaße ein Entscheidungsbaum erstellt, mithilfe dessen die Netzwerke schrittweise in quantitative Typen eingeteilt werden (siehe Abbildung 4.5b).

4. Schließlich werden die Zusammenhänge der gefundenen Netzwerktypen mit den Attributen der Egos untersucht.

Die Daten verlassen während dieser Prozedur immer wieder die vermeintlich hoch standardisierten und statistisch abgesicherten Bereiche und gehen durch die Forscher*innen hindurch - so z. B. bei der Auswahl eines geeigneten Plotting-Algorithmus sowie der zu plottenden Attribute oder bei der visuellen Typisierung der Netzwerke. Die Ergebnisse dieser Prozedur müssen daher als Produkt einer reflexiven Auseinandersetzung der Forschenden mit ihrem Datenmaterial betrachtet werden. Sie sind mit den darin zu Relevanz gekommenen Bedeutungszusammenhängen verschmolzen. Eine Trennung zwischen ,subjektiver" Deutung und „objektiver" Analyse lässt sich anhand des Analyseergebnisses nicht mehr vornehmen. Vermutlich würden Forschende mit unterschiedlichen Hintergründen und Fragestellungen die Netzwerke unter Anwendung derselben Methode auf verschiedene Weise typisieren.
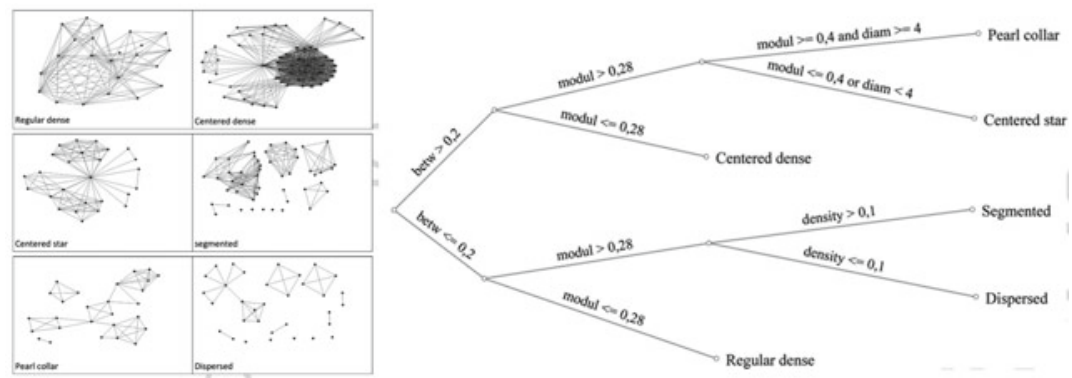

Abbildung 4.5 a) Typisierung; b) Entscheidungsbaum aus Bidart et al. 2018: 6/7 
Diese Vorgehensweise verstößt eindeutig gegen die oben genannten Gütekriterien quantitativer Forschung (Objektivität, Intersubjektivität, Wiederholbarkeit, Repräsentativität) während die Datengrundlage die Gütekritertien qualitativer Forschung verletzt ${ }^{13}$. Dennoch weisen die Ergebnisse eine gewisse Plausibilität auf. Anhand der Korrelationen zwischen Netzwerktyp und Ego-Attributen lassen sich am Ende sogar systematische Muster identifizieren, die statistischen Tests standhalten. Visualisierungen quantitativ erhobener Netzwerkdaten stehen daher an der Schnittstelle zwischen qualitativer und quantitativer Datenerhebung und -analyse (Molina et al., 2014, S. 306; Tubaro et al., 2016, 4 f.). Sie können auf verschiedene Weise bei der Datenanalyse eingesetzt werden (Molina et al., 2014, 328 ff.):

- als theoriegenerierendes Instrument zur Suche nach Strukturen und Clustern sowie nach Einflussgrößen auf die Strukturierung von Netzwerken,

- zur Visualisierung und Plausibilisierung quantitativer und qualitativer Analysen oder

- zur Validierung von Ergebnissen.

Darüber hinaus lassen sich quantitative Netzwerkdaten und -visualisierungen bereits bei der Datenerhebung im Rahmen sogenannter Fully Integrated Mixed Methods Designs einsetzen (Hollstein, 2014, 15 f.; Hollstein et al., 2020, Beispiele finden sich bei Avenarius \& Johnson, 2014; Noack \& Schmidt, 2013; Tubaro et al., 2016). Davon wurde auch in der vorliegenden Studie Gebrauch gemacht, indem die Interviewten bei der quantitativen Netzwerkabfrage gebeten wurden, die genannten Personen und Relationen zu erläutern. Diese Erzählungen wurden in den qualitativen Datensatz mit aufgenommen (transkribiert). An dieser Stelle verschmolzen die qualitativen und quantitativen Erhebungsmethoden zu einem integrierten Design.

Ziel dieses Exkurses war die methodologische Einordnung der in dieser Arbeit verwendeten Datengrundlage und -analyse. Dabei kommen quantifizierende und narrative Daten zur Struktur und Bedeutung sozialer Beziehungen zum Einsatz. Beide Datenquellen für sich ergeben nur ein unvollständiges Bild der sozialen Einbettung der Befragten. Erst in der Kombination der beiden Analysemethoden

\footnotetext{
${ }^{13}$ sofern es so etwas überhaupt gibt: Die einen plädieren für eine allgemeine Bestimmung von Gütekriterien wie Gegenstandsangemessenheit, empirische Sättigung, theoretische Durchdringung textuelle Performanz und Originalität (Strübing et al. 2018), die anderen bezeichnen die Definition von Gütekriterien qualitativer Forschung als Festlegung eines Mainstreams, der unkonventionelle Ansätze exkludiert (Eisewicht und Grenz 2018, Reichertz 2019).
} 
offenbart sich der Mehrwert dieser Vorgehensweise: quantitative Strukturanalysen bilden ab, aus welchen Akteur*innen die egozentrierten Netzwerke im Sample zusammengesetzt sind und wie die Beziehungen zwischen diesen Personen verteilt sind; die qualitativen Daten bereichern den strukturellen Überblick um Erkenntnisse darüber, wie die Netzwerke von den Befragten wahrgenommen werden, welchen Dynamiken sie unterliegen und welchen Einfluss sie auf das Handeln der Befragten ausüben.

Open Access Dieses Kapitel wird unter der Creative Commons Namensnennung 4.0 International Lizenz (http://creativecommons.org/licenses/by/4.0/deed.de) veröffentlicht, welche die Nutzung, Vervielfältigung, Bearbeitung, Verbreitung und Wiedergabe in jeglichem Medium und Format erlaubt, sofern Sie den/die ursprünglichen Autor(en) und die Quelle ordnungsgemäß nennen, einen Link zur Creative Commons Lizenz beifügen und angeben, ob Änderungen vorgenommen wurden.

Die in diesem Kapitel enthaltenen Bilder und sonstiges Drittmaterial unterliegen ebenfalls der genannten Creative Commons Lizenz, sofern sich aus der Abbildungslegende nichts anderes ergibt. Sofern das betreffende Material nicht unter der genannten Creative Commons Lizenz steht und die betreffende Handlung nicht nach gesetzlichen Vorschriften erlaubt ist, ist für die oben aufgeführten Weiterverwendungen des Materials die Einwilligung des jeweiligen Rechteinhabers einzuholen.

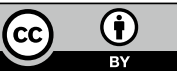

